\begin{tabular}{ccc}
\hline & & 0 \\
0 & International Journal of Case Reports \\
(ISSN:2572-8776)
\end{tabular}

\title{
Early effectivity of teduglutide for the treatment of short bowel sydrome - our first clinical experience
}

\section{Gombošová Laura1, Krivuš Juraj², Lazúrová Ivica1, Dedinská Ivana, Mokáň Marián²}

${ }^{1}$ internal clinic of University hospital L.Pasteur and Faculty of Medicine of P.J. Šafárik University, Košice, Slovakia; ${ }^{2}$ internal clinic of University Hospital of Martin and Jessenius Faculty of Medicine in Martin, Comenius University, Bratislava, Slovakia; ${ }^{3}$ Transplant center of University Hospital of Martin and Jessenius Faculty of Medicine in Martin, Comenius University, Bratislava, Slovakia

\section{ABSTRACT}

\section{Background}

Short bowel syndrome is an uncommon malabsorptive condition most frequently caused by massive surgical resection of the small intestine. Standard of care is home parenteral nutrition. Teduglutide, glucagon like peptide 2 analog, was approved to treat patients with short bowel syndrome, who are stable following a period of post-surgery intestinal adaptation.

\section{Case presentation}

We report two cases of patients with short bowel syndrome on home parenteral nutrition, treated with teduglutide, novel advanced therapy. We monitored the effectiveness and tolerance of the new treatment.

\section{Conclusion}

Teduglutide increases intestinal absorption, causes cryptal hyperplasia, villous hypertrophy, angiogenesis, and allows weaning from parenteral nutrition. We confirmed the early onset of treatment effectiveness in the 4th month. Early effectiveness of treatment allowed a reduction in the volume of home parenteral nutrition with an improvement in quality of life.

Keywords: short bowel syndrome, teduglutide, glucagon like peptide 2, home parenteral nutrition.
${ }^{*}$ Correspondence to Author:

Krivuš Juraj MD

University Hospital, Kollárova 2, 03601 Martin, Slovakia.

Tel: +421904248300

How to cite this article:

Gombošová Laura, Krivuš Juraj, Lazúrová Ivica, Dedinská Ivana, Mokáň Marián. Early effectivity of teduglutide for the treatment of short bowel sydrome - our first clinical experience. International Journal of Case Reports, 2021, 5:230

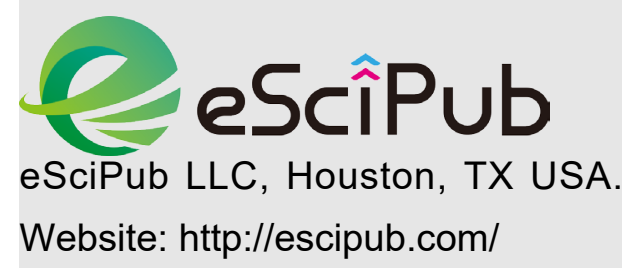




\section{Introduction}

Short bowel syndrome [SBS] is an uncommon malabsorptive condition most frequently caused by massive surgical resection of the small intestine [due to Crohn's disease, mesenteric ischemia, adhesive ileus or traumatic conditions]. SBS is associated with significant morbidity and mortality, reduced quality of life, and high healthcare costs. [1]. The clinical manifestations are only partially related with the length of the resected intestine. It depends on the abillity of the residual gastrointestinal tract to compensate the resected part and to adapt functionally to the new anatomical condition [2]. SBS belongs to intestinal failure, defined as the reduction of the gut function below the minimum necessary for the absorption of macronutrients and/or water and electrolytes. [2]. Home parenteral nutrition [HPN] is the primary lifesaving therapy for patients with chronic IF due to benign and malignant diseases [3]. Chronic IF treated with HPN may be associated with lifethreatening complications of either the underlying disease or HPN, the latter accounting for around $14 \%$ of total deaths [such as venous catheter-related complications and intestinal failure associated liver disease]. [4]. Novel therapy of SBS is teduglutide, recombinant analog of enteral hormone glucagon-like peptide 2. It promotes intestinal growth, facilitates natrium recirculation, considered the primary mechanism by which many nutrients such as sugars and amino acids are absorbed. Therapy with teduglutide allows weaning from HPN with enteral autonomy onset ${ }^{[5]}$. In a phase-III trial on adults with SBS dependent on HPN, a significantly greater proportion of patients taking $0.05 \mathrm{mg} / \mathrm{kg} /$ day teduglutide than placebo recipients achieved $a \geq 20 \%$ reduction in weekly HPN volume from baseline to week 20 and maintained it to week $24^{[6]}$. Teduglutide is contraindicated in patients with active gastrointestinal malignancy, including those with nongastrointestinal malignancies. Colonoscopic examination and removal of any colorectal polyps is recommended within 6 months prior to initiation of teduglutide. Subsequently, surveillance colonoscopy is recommended at the end of 1 year and then every 5 years or more frequently as needed ${ }^{[7]}$.

\section{Case presentations}

Patient information, the 1. case

Patients history: 54-year-old woman without a relevant medical history suffered a car accident in 2016. The result was severe destructive injury to the skeleton and abdominal organs. She underwent surgery with small and large bowel resection [ileostomy with no colon-in-continuity was constructed, I.type of short bowel syndrome]. The rest of the small intestine is about $60 \mathrm{~cm}$, resection of the large intestine with the rest $40 \mathrm{~cm}$, blinded.

We indicated HPN and hydratation: all in one nutrition bag with $1710 \mathrm{kcal} / 1075 \mathrm{ml}$ for 10 hours daily in the week [7/7], $1000 \mathrm{ml}$ of balanced salt solution $7 / 7$ via Hickmans catheter and medication to slow the passage through the digestive tract and reduce the secretion of digestive juices.

The woman experienced several complications in a short time. The first one was thrombosis of un upper hollow vein and the internal jugular vein, in January 2017. The catheter had to be extracted and immediately inserted into the femoral vein, low molecular heparin treatment was recomended. After partial recanalization of the affected vessels Hickmans catheter was inserted into the left subclavian vein. Next complication was recurrent catheter sepsis [blood cultivation for Staphylococcus aureus], with preserved catheter treated with amoxyciline clavulanate, in 2018. Within two years, there was a partial adaptation of the intestine with reduced infusions form $7 / 7$ to $5 / 7$. In 2020, patient developed spondylodiscitis leading to the need to increase HPN to $7 / 7$ plus $5 / 7$ rehydration therapy.

Indication for teduglutide: after the antibiotic therapy of spondylodiscitis was patient indicated for the treatment with teduglutide with daily dose $0.26 \mathrm{ml}$ [2,6 mg] subcutaneously. In Slovakia 
was drug registered and available for therapy in November 2020. During therapy, we noted the prolapse of the stoma without further progression. The patient complains of fatigue, anorexia, bloating, stomach hardening. After changing the dosing time to the evening, the patient has no problems. After 20 weeks of treatment, we gradually reduced HPN more than $35 \%$ and total parenteral substitution by $25.6 \%$. Patient requires HPN only 3 times a week rehydratation support as she needed. The patient's is laboratory results are stabile.

Outcomes of treatment: after 20 weeks, we evaluated the treatment as effective. At 40.th week of treatment, the patient could be completely weaned from parenteral support. Oral feeding was effective. The patient's quality of life has significantly improved. She is not dependent on infusions, she can eat normally.

\section{Case presentation}

Patient information, the 2. case

Patients history: 59 years old man with negative medical history was suffered from adhesive ileus, in 2017 year. Histological examination confirmed fibrinous purulent visceral peritonitis of unclear etiology. The condition required urgent surgical treatment, subtotal intestinal resection [60 $\mathrm{cm}$ rest of small intestine] with right sided hemicolectomia was performed. Type II. short bowel syndrome has developed, without ostomy, with colon in continuity.

Home parenteral nutrition $1500 \mathrm{kcal}$ together with $1000 \mathrm{ml}$ of balanced solutions was started. Parenteral nutrition with oral hyperphagia 2500 kcal daily was combined, with metabolic and water homeostasis, without complications, without weight loosing. After two years [in 2019] recurrent catheter-related sepsis was diagnosed [Stafylococcus aureus]. Sepsis was treated with antibiotics and catheter replacement. The patient works full time and HPN with daily infusion regime is limiting for him.

Indication for teduglutide therapy: treatment with GLP2 analog was indicated [in the time of teduglutide registration in our country, in
November 2020]. Screening colonoscopy is a mandatory examination before teduglutide treatment. Colonoscopy revealed tubulovilous adenomatous polyp, endoscopic polypectomy was performed. Teduglutide dose adjusted to weight was $0,32 \mathrm{ml} / 3,2 \mathrm{mg}$ once daily, subcutaneously. Abdominal pain and bloating occurred during the first month of treatment, with resolution later. In the fifth month of treatment we registered a reduction in diarrhea [from 7 to 3 bowel movements], stool thickening, increased diuresis without oedemas.

Outcomes of treatment: Reduction of the volume of parenteral substitution was possible from $7 / 7$ to $4 / 7$ [43\% reduction] with balanced water and mineral homeostasis. We anticipate further reduction of parenteral support with possible weaning from HPN and improving the quality of life. Also in this patients we evaluate the treatment as effective. At 28 weeks of treatment, it was possible to further reduce the parenteral support to 3 infusions per week [regime 3/7, 58\% reduction in parenteral substitution from the start of HPN]. It is assumed that it will be possible to terminate parenteral feeding in a short time.

\section{Discussion}

Teduglutide, glucagon like peptide 2 analog, was approved to treat patients with short bowel syndrome, who are stable following a period of post-surgery intestinal adaptation Teduglutide has demonstrated clinical effectiveness in realworld studies. Our first clinical experiences confirms the onset of early effectiveness of treatment, with mild self limited adverse events at the beginning. Both patients reported stool thickening, reduced bowel movements [reduced stoma output], loss of thirst, and increased diuresis. According real life experiences improved stool consistency and frequency, as well as the sensation of thirst, were identified as early clinical markers of response. [8]. Already in the fifth month of treatment it was possible to reduce parenteral substitution by more than $20 \%$. Finaly, in the 40 .th week 1. pactient weaned from parenteral substitution. Our results are consistent with real world data. In a single- 
centre, retrospective study of anatomically heterogeneous patients with SBS-IF 15 of 19 [79\%] achieved a decrease of at least $20 \%$ in parenteral support volume with teduglutide after treatment durations varying between 1 and 45 weeks. 2 of these patients [11\%] obtained complete independence from PS. Reductions in parenteral support requirements were accompanied by significant increases in mean villus height and crypt depth in this cohort as assessed by direct measurement of biopsies obtained during endoscopy [9]. HPN volume reductions were associated with improvements in quality of life scores for patients with short bowel syndrome treated with teduglutide [10]. During further treatment, we anticipate weaning our patients from HPN. This is a great advantage for a full time working patient. We preliminarily assess that teduglutide treatment is effective and safe for patients with short bowel syndrome. Hypertrophy of ostomy was not progressed. Integrated safety data for 222 person-years of exposure to teduglutide in four clinical trials demonstrated that teduglutide has a safety profile in adult patients with SBS-IF consistent with prior studies. [9]. Teduglutide was associated with a significantly increased risk of gastrointestinal stoma complication compared with placebo. Stoma enlargement was observed in clinical trials and in real-world clinical practice [9]. The strength of the work is that this is the first experience in our country, with very good treatment effectiveness. Our experience will contribute positive information to the real world evidence data. The limitation of work is the small number of treated patients.

Take away lesson from these cases - GLP 2 analog treatment in our first case resulted in early weaning from home parenteral nutrition [during 10 months] and in second case resulted in $58 \%$ reduction of HPN during 7 months. Teduglutide will allow a significant improvement in quality of life, without dependence on parenteral nutrition.

\section{Conclusion}

Treatment for short bowel syndrome is well advanced. An innovative treatment is teduglutide, which allows weaning from parenteral nutrition and relieves the patient of a large number of infusion bags and medical devices. In practice, we have confirmed the early onset of effectiveness of GLP2 treatment. Therapy is well tolerated, with mild adverse events [abdomen pain] in the start of therapy. Significantly improves the quality of life of patients.

\section{Conflict of interests}

Authors declare no conflicts of interest.

\section{Availability of data and materials}

All data supporting the findings of this article are included in the manuscript.

\section{Abbreviations:}

SBS - short bowel syndrome

HPN - home parenteral nutrition

IF- intestinal failure

$7 / 7$ - administration of i.v. nutrition 7 days a week

\section{Ethics declarations}

Ethics approval and consent to participate. Not applicable.

\section{Consent for publication}

Written informed consent was obtained from the patient's legal guardians for publication of this case report and any accompanying images. A copy of the written consent is available for review by the Editor-in-Chief of this journal.

\section{Competing interests}

The authors declare that they have no competing interest to disclose.

Acknowledgements: Not applicable

Funding: No funding was secured for this study.

\section{Contributions}

LG and JK performed the data collection. LG drafted the initial manuscript. JK, ID reviewed and $\mathrm{IL}$ revised the manuscript. ID and MM contributed to the conception of the manuscript and interpretation of the data, critically reviewed and revised the manuscript. IL coordinated and 
supervised data collection, reviewed and revised the manuscript. All authors took care of the patient and approved the final manuscript as submitted and agree to be accountable for all aspects of the work. All authors read and approved the final manuscript.

\section{References}

[1]. Massironi S, Cavalcoli F, Rausa E, Invernizzi P, Braga M, Vecchi M. Understanding short bowel syndrome: Current status and future perspectives. Dig Liv Dis. 2020; 52: 253-261.

[2]. Pironi L, Arends J, Baxter J, Bozzetti F, Peláez $R B$, Cuerda $C$, et al. ESPEN endorsed recommendations. Definition and classification of intestinal failure in adults. Clin Nutr. 2015; 34:171- 80.

[3]. Pironi L, Arends J, Bozzetti F, Cuerda C, Gilland ers $L$, Jeppesen PB, et al. ESPEN guidelines on chronic intestinal failure in adults. Clin Nutr. 2016; 35[2]: 247-307.

[4]. Pironi L, Boeykens K, Bozzeti F, Joly F, Klek S, Lal $S$, et al. ESPEN guideline on home parenteral nutrition. Clin Nutr. 2020; 1645-1666.

[5]. Brubaker PL, Why Recycling Matters: Glucagon-Like Peptide-2 and the Regulation of Intestinal Sodium and Fluid Absorption. Dig Dis Sci. 2020; 65:3422-3424.

[6]. Jeppessen PB, Pertkiewitz M, Messing B, lyer K, Seidner DL, O'Keefe SJ, et al. Teduglutide reduces need for parenteral support among patients with short bowel syndrome with intestinal failure. Gastroenterol. 2012; 143[6]:1473-1481.

[7]. Vipperla K, O'Keefe SJ. Targeted therapy of short-bowel syndrome with teduglutide: the new kid on the block. Clin Exp Gastroenterol. 2014; 7: 489-495.

[8]. Schoeler M, Klag T, Wendler J, Bernhard S, Adolph M, Kirschniak A, et al. GLP-2 analog teduglutide significantly reduces need for parenteral nutrition and stool frequency in a reallife setting. Ther Adv Gastroenterol 2018; 11: 111.

[9]. Pape UF, lyer KR, Jeppesen PB, Kunecki M, Pironi L, Schneider SM et al. Teduglutide for the treatment of adults with intestinal failure associated with short bowel syndrome: pooled safety data from four clinical trial. Ther Adv Gastroenterol. 2020; 13: 1-18.

[10]. Jeppesen PB, Pertkiewicz M, Forbes A, Pironi L, Gabe SM, Joly F et al. Quality of life in patients with short bowel syndrome treated with the new glucagon-like peptide-2 analogue teduglutide--analyses from a randomised, placebo-controlled study. Clin Nutr. 2013; 32 [5]:713-21. 